\title{
Universality of Anderson transition in two-dimensional systems of symplectic symmetry class
}

\author{
Reza Sepehrinia \\ School of Physics, Institute for Research in Fundamental Sciences, IPM, 19395-5531 Tehran, Iran
}

\begin{abstract}
We investigate localization of noninteracting particles with spins higher than $\frac{1}{2}$ in a twodimensional random potential in presence of spin-orbit coupling. We consider an integer spin $(s=1)$ and a half-integer spin $\left(s=\frac{3}{2}\right)$ belonging to orthogonal and symplectic symmetry classes, respectively. We show that particles with integer spin are localized and those with half-integer spin exhibit Anderson transition. The transition belongs to universality class of conventional symplectic model for spin- $\frac{1}{2}$ particles.

PACS numbers: 72.15.Rn, 71.70.Ej, 05.45.Df
\end{abstract}

\section{INTRODUCTION}

Symplectic class has a rich physical content among the symmetry classes in the Wigner-Dyson classification of random matrices. Spin-orbit scattering provides the common physical realization of this class. From the symmetry point of view such system is invariant under time reversal $(\mathcal{T})$ but not under spin rotation $(\mathcal{S})$. For a system with non-integer spin we have $\mathcal{T}^{2}=-1$ and the wave function (WF) has a rotational periodicity of $4 \pi$ therefore in average, time-reversed paths in the multiple-scattering picture interfere destructively. ${ }^{1}$ As a result of the destructive interference, conductivity is enhanced and we have weak anti-localization rather than weak localization. This is one of the mechanisms of criticality in two spatial dimensions which is specific for symplectic class in the framework of early Wigner-Dyson classification.

Several other realizations of symplectic class have been identified which exhibit distinct universal behavior. For this class the homotopy group of $\sigma$ model manifold is nontrivial so the $\sigma$ model action allows for inclusion of a topological $\theta$ term (see Ref. 2 for a recent review). Such topological term is responsible for quantum Hall criticality in the unitary class. Spin-orbit coupling have provided similar topological phases in the $\mathcal{T}$-invariant systems which has been the subject of an intense activity in recent years. ${ }^{3}$ Remarkably, quantum spin Hall (QSH) which is a novel phase induced by $Z_{2}$ topological term in the symplectic ensemble. A new universality class of Anderson transition emerges in presence of this topological structure between the metallic and QSH phases. ${ }^{4}$ It should be mentioned however that the corresponding Chalker-Coddington network model which allows to have odd number of Kramers doublets (i.e., nontrivial topology) does not capture this critical behavior. ${ }^{5}$ Another realization of symplectic class with nontrivial topology appears in a two-dimensional system of Dirac fermions $\mathrm{s}^{6,7}$ which yields a unconventional scaling $\beta$ function. Two different scenarios are proposed one of which predicts an extra attractive fixed point in the strong-coupling limit ${ }^{6}$ and the other one implies on delocalization of all states even in strong disorder limit. ${ }^{7}$ Based on semiclassical arguments it is also shown that spin-orbit scattering may induce a novel universality class in the regime of integer quantum-Hall effect. ${ }^{8}$ Whereas in the unitary ensemble, presence or absence of spin-rotational invariance does not change symmetry class. These examples imply the fact that in spite of a complete mathematical classification of symmetry classes, universality classes are not recognized so far.

Appreciate to new advances in designing periodic potentials by standing waves of light, many experiments which are not possible to arrange for electrons can be simulated with quantum motion of cold atoms. Especially it just recently became possible to do careful experiments on localization of noninteracting matter waves. Even more fascinating games could be done by changing the polarization of the beams. So the internal degrees of freedom of the atom can be coupled to the momentum of the beam and produce an effective spin-orbit like term in the Hamiltonian. Once this could be done, one can search for new universalities and topological properties of WFs with higher tunability. ${ }^{9}$

A natural generalization in this direction is to consider atoms with higher number of internal degrees of freedom or particles with higher spins. Here we want to address whether higher spins in spin-orbit interaction can change the universality class of transition or not. We use the transfer-matrix method to calculate the localization length and then extract the critical exponents from finite-size scaling analysis. On the other hand multifractal spectrum of critical WFs are of universal properties hence useful to describe the transition. We will examine multifractal properties of higher spin model in comparison with spin- $\frac{1}{2}$ case.

\section{MODEL}

Several models have been proposed to study Anderson localization problem in presence of spin-orbit scattering. ${ }^{10}$ Regardless of microscopic details, they present the same universal features. Spin-relaxation length is an important irrelevant length scale in these systems. $\mathrm{SU}(2)$ model $^{11}$ has smaller spin-relaxation length since spin rotation operators in each link of its lattice, 
are uniformly distributed. As a result it needs small correction to scaling. Having small size effects this model provides more accurate calculation of critical exponents. Here we use the generalization of this model to describe particles with higher spins. We start with the following Hamiltonian which is proposed for spin- $\frac{1}{2}$ particles.

$$
H=\sum_{i \sigma} \epsilon_{i} c_{i \sigma}^{\dagger} c_{i \sigma}-V \sum_{\langle i j\rangle \sigma \sigma^{\prime}} \mathcal{R}_{\sigma \sigma^{\prime}}^{i j} c_{i \sigma}^{\dagger} c_{j \sigma^{\prime}}
$$

Here we will let the hopping matrices act on spinors of higher rank. So spin indexes $\left(\sigma, \sigma^{\prime}\right)$ take the values $(s, s-1, \cdots,-s)$ for particles with spin $s$. Latin indices denote nearest-neighbor sites on square lattice. Random on-site potential $\epsilon_{i}$ is distributed uniformly in the interval $\left[-\frac{W}{2}, \frac{W}{2}\right]$. Energy scale will be set by $V=1$.

$\mathcal{R}^{i j}$ 's are $(2 s+1)$-dimensional irreducible representation of $\mathrm{SU}(2)$ group. In terms of Euler angles they have the following description $\mathcal{R}_{\sigma \sigma^{\prime}}^{i j}=$ $D_{\sigma \sigma^{\prime}}^{s}\left(\alpha_{i j}, \beta_{i j}, \gamma_{i j}\right)=e^{i\left(\sigma \alpha_{i j}+\sigma^{\prime} \gamma_{i j}\right)} d_{\sigma \sigma^{\prime}}^{s}\left(\beta_{i j}\right), \quad$ where $d_{\sigma \sigma^{\prime}}^{s}\left(\beta_{i j}\right)=\left\langle s \sigma\left|e^{-i \beta_{i j} S_{y} / \hbar}\right| s \sigma^{\prime}\right\rangle$ is the matrix element of rotation operator around $y$ axis. Angles $\alpha, \beta$, and $\gamma$ are distributed randomly in different links of lattice such that rotation matrices $\mathcal{R}^{i j}$ have uniform distribution with respect to the Haar measure on $\mathrm{SU}(2)$ group. Namely, $\alpha$ and $\gamma$ are distributed uniformly in the interval $[0,2 \pi)$ and $\beta$ is chosen from interval $\left[0, \frac{\pi}{2}\right]$ with distribution $P(\beta)=\sin (2 \beta)$.

\section{LEVEL STATISTICS}

Regarding broken spin-rotational symmetry of Hamiltonian (1), for integer spins it does belong to the orthogonal ensemble. Only for half-integer spins it falls into the symplectic ensemble. This is known from Wigner-Dyson classification of random matrices. ${ }^{12}$ An essential difference in the spectrum of two cases is Kramers degeneracy of energy levels of later which is robust against disorder.

In this section we demonstrate above-mentioned relation with the Wigner-Dyson symmetry classes numerically. The simplest quantity which can be used to determine the statistical properties of energy levels is the nearest level-spacing distribution. To have comparable results with the distributions of random matrix theory we look at the distribution of $\delta_{n}=\frac{E_{n+1}-E_{n}}{\left\langle E_{n+1}-E_{n}\right\rangle}$ which is unfolded level spacing. Denominator is the ensemble average of level spacing which is proportional to inverse density of states (DOS). So unfolding procedure is needed when the DOS has large variations within the energy range under consideration. Obviously firstorder moment of distribution function $P(\delta)$ is fixed, i.e., $\langle\delta\rangle=\int_{0}^{\infty} \delta P(\delta) d \delta=1$ for unfolded spectrum. We determine the distribution function $P(\delta)$ for two $s=1$ and $s=\frac{3}{2}$ cases in the metallic regime, after removing the degeneracy in the later case. By metallic regime we mean weak disorder for which WFs have large overlap and comparable localization length with the system size. We find

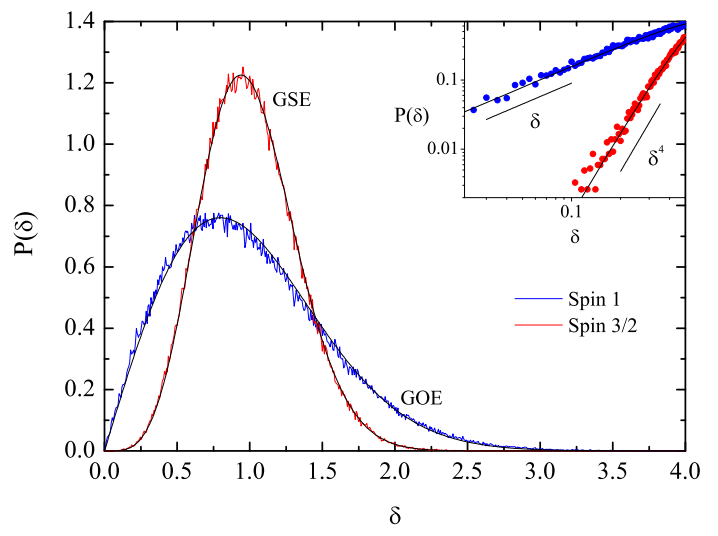

FIG. 1: (Color online) Level-spacing distribution function for disorder strength $W=0$. Spin $1(\mathrm{GOE})$, spin $\frac{3}{2}$ (GSE) and corresponding Wigne-Dyson distributions with $\beta=1$, 4 , respectively. Inset: log-log plot which shows power-law behavior at $\delta \rightarrow 0$. Line segments represent functions proportional to $\delta$ and $\delta^{4}$.

good agreement with GOE $(\beta=1)$ for $s=1$ and GSE $(\beta=4)$ for $s=\frac{3}{2}$ cases. The results which are shown in Fig. 1 are obtained by diagonalizing $10^{3}$ Hamiltonians of lattice size $20^{2}$ and disorder width $W=0$. For nonzero but small values of $W$ also we obtain the same results. In the strong disorder limit which all states (for both cases) tend to be localized one naturally expect to see Poisson distribution. Difference between two cases would reveal in the intermediate disorder strength.

\section{TRANSFER MATRIX}

To find a precise insight in to the localization properties of these models and to explain the differences in the thermodynamic limit we implement a finite-size scaling analysis. In the following we will study renormalized localization length (RLL), $\Lambda=\frac{\lambda_{m}}{M}$, on the quasione-dimensional geometry, where $\lambda_{m}$ is the localization length on strip. We utilize transfer-matrix method ${ }^{13}$ to calculate the minimal Lyapunov exponent, inverse of which is the largest length scale of spatial extension of wave function. Dimension of transfer matrices for spin $s$ is $N=2(2 s+1) M$ with $M$ being the system size in the transverse direction. Lyapunov exponents appear in $(-\gamma, \gamma)$ pairs for integer spin case due to symmetry of transfer matrices. Furthermore for halfinteger spin case each $\gamma$ appears twice due to Kramers degeneracy. According to these symmetries we need to evolve $\frac{N}{2}=(2 s+1) M$ vectors of length $N$ to calculate minimum positive $\gamma$. Components of vectors are $V_{(2 s+1) j+m}=\psi_{\sigma}^{n, j}, V_{(2 s+1)(j+M)+m}=\psi_{\sigma}^{n-1, j}$, where $n$ is the number of layer (here a chain of length $M$ ), $j=0, \ldots, M-1$ is the coordinate in the transverse direction and $m$ takes values $1, \ldots,(2 s+1)$ corresponding to $\sigma=s, s-1, \ldots,-s$, respectively. Gram-Schmidt orthogonalization is implemented after each four steps. 

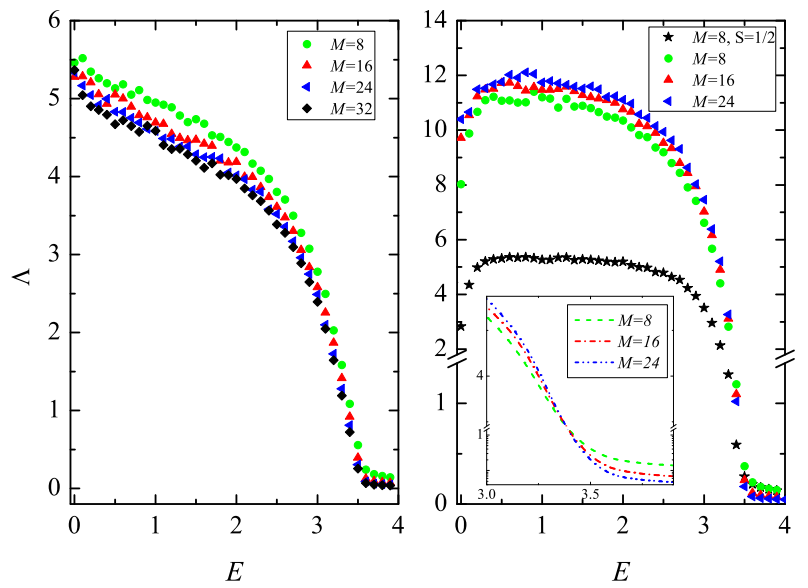

FIG. 2: (Color online) Left: RLL as a function of energy of spin-1 particle with zero on-site disorder $(W=0)$, right: spin $\frac{3}{2}$ and spin $\frac{1}{2}$ (for single size $M=8$ ) with $W=1$. Inset shows a zoom in around the crossing point.

Let us start with spin-1 particle and zero on-site disorder $(W=0)$. In the left panel of Fig. 2 we observe that $\Lambda$ decreases by increasing the size $M$ in the whole energy range. We can conclude that states are localized even for zero on-site disorder. In other words the randomness in spin rotation in passing through different links is enough to localize the particle. Results for nonzero on-site disorder are the same and we will not present them here. This is what we expect for a system in orthogonal (AI) symmetry class. That breaking of spin-rotational symmetry in a $\mathcal{T}$-invariant system with integer spin neither changes symmetry class nor develops delocalized states. We should comment on the additional symmetry which Hamiltonian (1) may have in absence of on-site disorder. Using periodic boundary conditions in the transverse direction and even $M$ the lattice will be a bipartite lattice which is shown to have anomalies at zero energy. ${ }^{14-16} \mathrm{We}$ can see in Fig. 2 that at the center of energy band, $\Lambda$ remains almost constant for different sizes which indicates a critical state at $E=0$. Away from the band center $\Lambda$ decreases more rapidly by increasing $M$, which gradually leads in creation of a cusp at $E=0$. For an odd number of channels $(M)$ with free boundary conditions in transverse direction particularly $\Lambda \rightarrow \infty$ for this zero mode. This is proved ${ }^{15}$ analytically for coupled one-dimensional chains with $\beta=1,2$. This critical state will be ruined by addition of small on-site disorder which breaks sublattice symmetry of Hamiltonian. Unlike the spin-1 particle the case with spin- $\frac{3}{2}$ possesses a band of extended states for certain values of on-site disorder strength $W$. As an example, results of $\Lambda$ for spin- $\frac{3}{2}$ particle with disorder strength $W=1$ and sizes $M=8,16,24$ are shown in Fig. 2. The result for spin- $\frac{1}{2}$ particle is also included for comparison at the same on-site disorder strength. Relative errors of data are $1 \%$ for $M>8,0.8 \%$ for $M=8$, and $0.7 \%$ for spin- $\frac{1}{2}$ case. To reach this accuracy the length of strips is increased up to $1.3,1.6,2.4,3.6 \times 10^{6}$

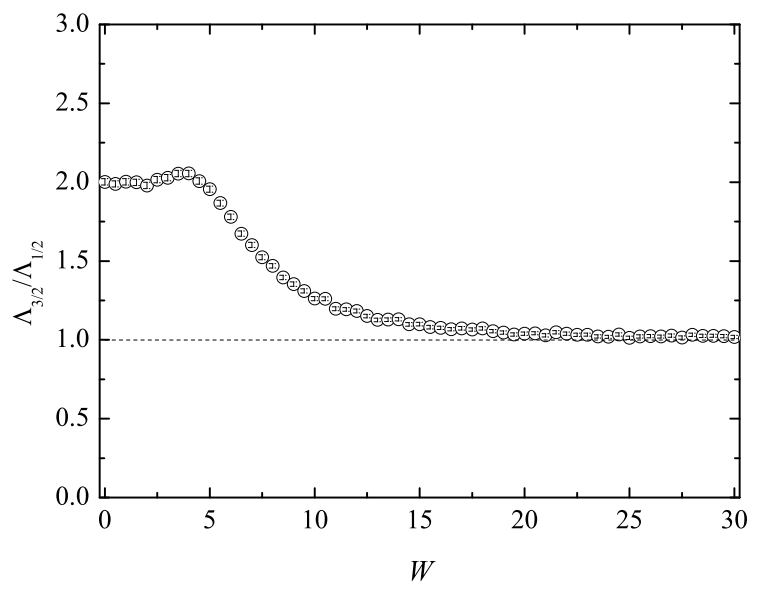

FIG. 3: Ratio of RLLs vs $W$ of spin- $\frac{3}{2}$ and spin- $\frac{1}{2}$ particles for single energy $E=2$ and $M=8$.

for spin 1 with $M=8,16,24,32,1.4,1.8,2.8,3.8 \times 10^{6}$ for spin $\frac{3}{2}$ with $M=8,16,24,32$, respectively, and $0.9 \times 10^{6}$ for spin- $\frac{1}{2}$ case. There can be seen a mobility edge at $E \approx 3.4$ where order of symbols is reversed. As usual the states in the band edge are localized and the midband states are extended. The finite band of the bulk extended states make the true metallic phase happen in the symplectic class. By increasing the disorder strength the band of extended states gets narrower and gradually collapses at a critical value $W_{c}$.

It is worthwhile to compare RLL of spin- $\frac{3}{2}$ and spin$\frac{1}{2}$ cases more closely. The ratio of RLLs vs $W$ of two models is plotted in Fig. 3 for single energy $E=2$ and $M=8$. The energy is chosen away from the band center and edges to ensure the DOS has considerable value in both models. In a range of weak disorder strengths $(W \lesssim 4)$, the ratio is nearly constant and equals 2 . This is where the hopping term is dominant or comparable with on-site term. In strong disorder limit $(W \gg 1)$ the hopping term is negligible, therefore spin degrees of freedom would not have considerable effect on localization length. Thus one expects the same RLL for both cases. That is what which can be seen also in Fig. 3 at large $W$. In the next sections we will discuss critical exponents characterizing the universality class of the transition.

\section{SCALING AND CRITICAL INDICES}

Dimensionless quantity $\Lambda$ is one of scaling variables which is frequently used for numerical analysis. On the basis of one-parameter scaling hypothesis, it can be written in the following form:

$$
\Lambda(E, W, M)=f\left(\frac{M}{\xi(W, E)}\right)
$$

where $\xi(W, E)$ is the localization length (insulating side) or the correlation length (metallic side) of infinite system. 
It is not the only length scale in this system. We will encounter deviations from scaling, Eq. (2), when other (irrelevant) length scales are comparable with correlation length. Near the mobility edge, $\xi$ diverges as $\xi \sim \mid E-$ $\left.E_{c}\right|^{-\nu}$ with critical exponent $\nu$. The value of $\Lambda$ at critical point is also a universal constant and independent of $W$.

To calculate the critical exponent we take few sets of data ( $\Lambda$ vs $E$ ) close to the mobility edge for $M=$ $8,16,24,32$. There are two ways of fitting the data to scaling form (2). Since function $f(x)$ is unknown we can either use a Taylor expansion and then obtain the coefficients by fitting, or take one set as $f(x)$ (by interpolating), define a suitable residual of curves and minimize it by adjusting critical indices. Let the values of $\Lambda$ and $E$ in the $i$ th set (corresponding to size $M_{i}$ ) be denoted by $\Lambda_{i j}$ and $E_{i j}$.

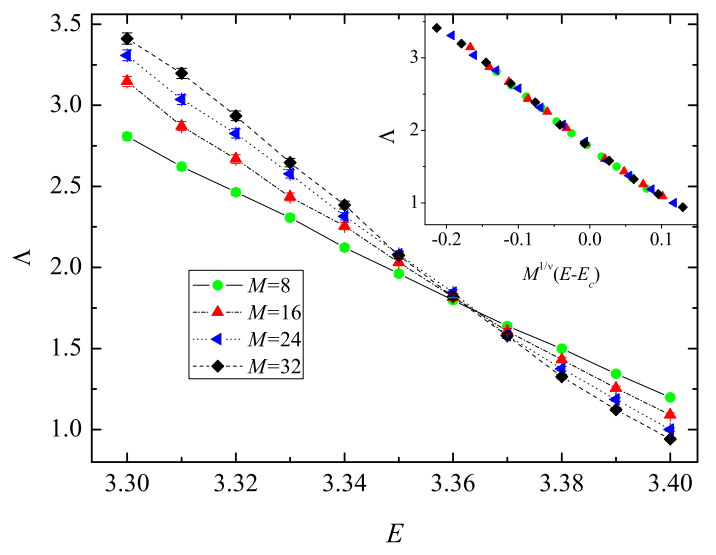

FIG. 4: (Color online) RLL of spin- $\frac{3}{2}$ particle for $W=1$ and sizes $M=8,16,24,32$. Inset: after rescaling.

A possible definition of residual follows ${ }^{17}$

$$
R=\frac{1}{N} \sum_{k} \sum_{i \neq k} \sum_{j}^{\prime}\left|\Lambda_{i j}-\Lambda_{k}\left(M^{1 / \nu}\left(E_{i j}-E_{c}\right)\right)\right|
$$

where $\Lambda_{k}(x)$ is obtained by transforming the horizontal axis of $\Lambda_{k j}$ such as $E_{k j} \rightarrow M_{k}^{1 / \nu}\left(E_{k j}-E_{c}\right)$ and simple linear interpolation. The prime on the third sum denotes summation over $j$ 's which are in the range of definition of $\Lambda_{k}(x)$ and $N$ is the total number of such points. For each $k$ one set is taken as the reference curve and the other curves are supposed to collapse on it by rescaling. Function $R$ reaches its minimum value $(\sim 0.01)$ at $\nu=$ $2.81 \pm 0.18$ and $E_{c}=3.362 \pm 0.014$. Error-bars are roughly estimated from width of minimum by using approximate expressions given in Ref. 17. Reasonable data collapse is obtained for these values of critical parameters (inset of Fig. 4).

We tabulate the values of critical exponents in Table I. The exponents of spin- $\frac{1}{2}$ system are also given for comparison. In spite of relatively larger error-bars in the spin- $\frac{3}{2}$ case, estimated exponent is close to that of spin- $\frac{1}{2}$ particle. Our speculation is that two models belong the same universality class.
TABLE I: Critical exponents: results of spin- $\frac{1}{2}$ case are taken from Ref. 11

\begin{tabular}{lcc}
\hline \hline Model & $\nu$ & $\Lambda_{c}$ \\
\hline Spin $\frac{1}{2}$ & $2.73 \pm 0.02$ & $1.844 \pm 0.001$ \\
Spin $\frac{3}{2}$ & $2.81 \pm 0.18$ & $1.77 \pm 0.07$ \\
\hline
\end{tabular}

\section{MULTIFRACTAL SPECTRUM}

Fluctuations of critical WFs exhibit universal features. So multifractal exponents which describe the distribution of WFs can be used to characterize phase transition. Moreover multifractality leads to anomalous diffusion of wave packets near the mobility edge. This is reflected in power-law decay of return probability which is governed by one of those multifractal exponents. We want to compare the multifractal spectrum of spin- $\frac{3}{2}$ and spin- $\frac{1}{2}$ particles at the mobility edge. Scaling properties of multifractal measure are encoded in the $f(\alpha)$ spectrum. Here we use the direct method of Chhabra and Jensen ${ }^{18,19}$ to calculate this function. By using the box probabilities $p(l)=\int_{\Omega(l)} d^{2} \boldsymbol{r}|\Psi(\boldsymbol{r})|^{2}$, with $\Omega(l)$ being a box of linear dimension $l$, and one-parameter families of normalized measures $\mu_{i}(q, l)=p_{i}^{q}(l) / \sum_{i} p_{i}^{q}(l)$ for each value of $q$, corresponding values of $\alpha(q)$ and $\tilde{f}(q)=f[\alpha(q)]$ can be calculated from

$$
\alpha(q)=\frac{\sum_{i} \mu_{i} \ln p_{i}}{\ln (l / L)}, \quad \tilde{f}(q)=\frac{\sum_{i} \mu_{i} \ln \mu_{i}}{\ln (l / L)} .
$$

In practice, linear fit to numerators vs $\ln (l / L)$ can be used to calculate $\alpha$ and $\tilde{f}$ as the slope of fitted line. This method requires large system sizes to avoid finite-size effect.

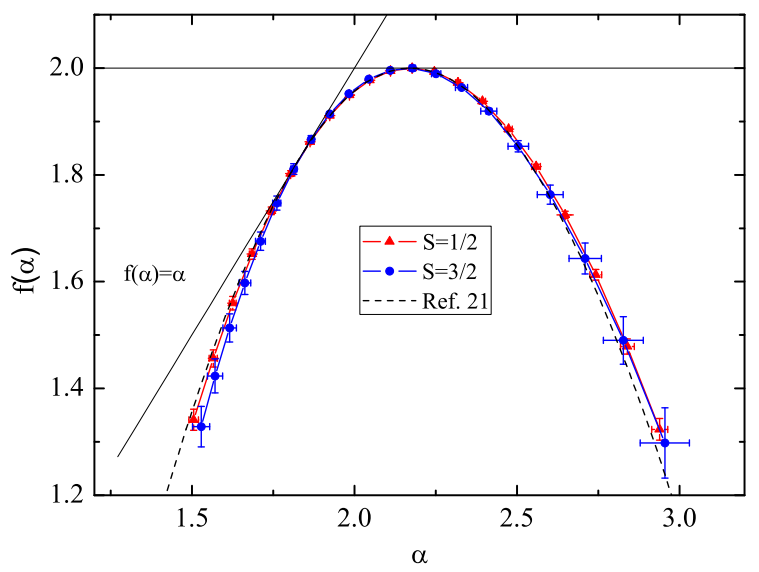

FIG. 5: (Color online) Singularity spectrum of critical WFs of spin- $\frac{1}{2}$ and spin- $\frac{3}{2}$ particles with lattice sizes $1500^{2}$ and $800^{2}$, respectively. Line $f(\alpha)=\alpha$ is tangent to $f(\alpha)$ curve as it should be. Horizontal line shows maximum of $f(\alpha)$ which is dimension of support $(d=2)$. The data from Ref. 21 are included for comparison.

These exponents are related to correlation dimension 
$\tau(q)$ by a Legendre transform $\alpha(q)=d \tau(q) / d q, f[\alpha(q)]=$ $\alpha(q) q-\tau(q)$ and generalized dimension $D(q)$ is defined through $\tau(q)=(q-1) D(q)$.

The singularity spectrum for single critical states of spin- $\frac{1}{2}$ and spin- $\frac{3}{2}$ particles are shown in Fig. 4. Corresponding lattice sizes are $1500^{2}$ and $800^{2}$, respectively. The eigenstates are obtained via Lanczos algorithm. Parameters of former is picked up from phase diagram obtained in Ref. $11(E=1, W=5.952)$ and the spin- $\frac{3}{2}$ case has $(E=3.362, W=1)$. Even thought two states are distant in parameter space, their singularity spectrum are the same within the error bars. Especially we obtain (for WFs in Fig. 4) $\alpha_{0}^{1 / 2}=2.174 \pm 0.005$ and $D^{1 / 2}(2)=$ $1.66 \pm 0.04$. These exponents and also whole spectrum are compatible with previous calculations based on inverse participation ratio analysis. ${ }^{20,21}$ For spin- $\frac{3}{2}$ case $\alpha_{0}^{3 / 2}=2.164 \pm 0.009$ and $D^{3 / 2}(2)=1.71 \pm 0.05$, where $\alpha_{0}=\alpha(0)$. Error bars are standard deviation of slopes in linear fitting (Fig. 5).

It should be noted that, obtained results depend weakly on realization of disorder so ensemble averaging will not change them significantly.

\section{SUMMARY AND FUTURE WORK}

Localization of particles with spin 1 and $\frac{3}{2}$ in the presence of spin-orbit interaction is studied numerically in the $\mathrm{SU}(2)$ model. In summary, spin-1 particle belongs to orthogonal symmetry class and is always localized. Spin- $\frac{3}{2}$ case exhibits a transition. Implication of finite-size scaling results and multifractal analysis on critical WFs of this model is that the transition belongs to the conventional universality in symplectic class.

An interesting direction of future work would be investigation of spin dependence of localization length (see Fig. 3) within the Dorokhov-Mello-Pereyra-Kumar formalism for disordered wires.

\section{ACKNOWLEDGMENTS}

It is pleasure to thank P. Markos for helpful discussions. We would like also to thank H. Obuse and his coworkers for sharing their data from Ref. 21, R. Nourafkan for providing his Lanczos code and F. Joibari for reading the manuscript.
1 G. Bergmann, Solid State Commun. 42, 815 (1982).

2 F. Evers and A. D. Mirlin, Rev. Mod. Phys. 80, 1355 (2008).

3 C. L. Kane and E. J. Mele, Phys. Rev. Lett. 95, 146802 (2005); D. N. Sheng, Z. Y. Weng, L. Sheng, and F. D. M. Haldane, ibid. 97, 036808 (2006); B. A. Bernevig, T. L. Hughes, and S.-C. Zhang, Science 314, 1757 (2006).

4 M. Onoda, Y. Avishai, and N. Nagaosa, Phys. Rev. Lett. 98, 076802 (2007).

${ }^{5}$ H. Obuse, A. Furusaki, S. Ryu, and C. Mudry, Phys. Rev. B 76, 075301 (2007).

6 P. M. Ostrovsky, I. V. Gornyi, and A. D. Mirlin, Phys. Rev. Lett. 98, 256801 (2007).

7 K. Nomura, M. Koshino, and S. Ryu, Phys. Rev. Lett. 99, 146806 (2007); J. H. Bardarson, J. Tworzydlo, P.W. Brouwer, and C. W. J. Beenakker, ibid. 99, 106801 (2007).

${ }^{8}$ Y. Avishai and Y. Meir, Phys. Rev. Lett. 89, 076602 (2002).

9 A. M. Dudarev, R. B. Diener, I. Carusotto, and Q. Niu, Phys. Rev. Lett. 92, 153005 (2004).

10 T. Ando, Phys. Rev. B 40, 5325 (1989); S. N. Evangelou, Phys. Rev. Lett. 75, 2550 (1995).

11 Y. Asada, K. Slevin, and T. Ohtsuki, Phys. Rev. Lett. 89,
256601 (2002).

12 M. L. Mehta, Random Matrices (Academic, Boston, 1991).

13 P. Markos, Acta Phys. Slov. 56, 561 (2006), and references therein.

14 J. Miller and J. Wang, Phys. Rev. Lett. 76, 1461 (1996).

15 P. W. Brouwer, C. Mudry, B. D. Simons, and A. Altland, Phys. Rev. Lett. 81, 862 (1998).

16 P.W. Brouwer, C. Mudry, and A. Furusaki, Phys. Rev. Lett. 84, 2913 (2000).

17 S. M. Bhattacharjee and F. Seno, J. Phys. A 34, 6375 (2001)

18 A. Chhabra and R. V. Jensen, Phys. Rev. Lett. 62, 1327 (1989).

19 For a review on multifractality in localizationdelocalization transition, see M. Janssen, Int. J. Mod. Phys. B 8, 943 (1994).

20 A. Mildenberger and F. Evers, Phys. Rev. B 75, 041303(R) (2007).

21 H. Obuse, A. R. Subramaniam, A. Furusaki, I. A. Gruzberg, and A.W.W. Ludwig, Phys. Rev. Lett. 98, 156802 (2007). 